\title{
Respiratory failure in a diabetic ketoacidosis patient with severe hypophosphatemia
}

\author{
Han Saem Choi, MD', \\ Ahreum Kwon, MD', \\ Hyun Wook Chae, MD', \\ Junghwan Suh ,MD', \\ Duk Hee Kim, MD, PhD', \\ Ho-Seong Kim, MD, PhD
}

'Department of Pediatrics, Severance Children's Hospital, Endocrine Research Institute, Yonsei University College of Medicine, Seoul, 'Sowha Children's Hospital, Seoul, Korea
Received: 20 September, 2017

Revised: 18 September, 2017

Accepted: 6 November, 2017

Address for correspondence: Ho-Seong Kim, MD, PhD

Department of Pediatrics, Severance Children's Hospital, Yonsei University College of Medicine, 50-1 Yonsei-ro, Seodaemun-gu, Seoul 03722, Korea

Tel: +82-2-2228-2069

Fax: +82-2-393-9118

E-mail:kimho@yuhs.ac

https://orcid.org/0000-0003-1135099X
Phosphate is essential in regulating human metabolic processes, and severe hypophosphatemia can induce neurologic and hematological complications and result in respiratory failure and cardiac dysfunction. Therefore, correction of severe hypophosphatemia can be pivotal in the management of diabetic ketoacidosis (DKA). We report the case of a 14-year-old female who was diagnosed with type 1 diabetes and referred to our institute for treatment of DKA. Although the patient received fluid and continuous insulin administration according to the current DKA treatment protocol, generalized tonic seizures and cardiac arrest developed. After cardiopulmonary resuscitation, the patient recovered and was stable. Within 16 hours after DKA treatment, the patient developed respiratory failure with severe hypophosphatemia that required mechanical ventilation. Concurrent neurologic evaluation revealed no specific abnormalities. The patient recovered without any complications after correcting the hypophosphatemia. We suggest vigilant monitoring of the phosphate level in DKA patients and active replacement when required.

Keywords: Diabetic ketoacidosis, Hypophosphatemia, Respiratory failure

\section{Introduction}

Diabetic ketoacidosis (DKA) is a life-threatening acute complication of diabetes mellitus. DKA is characterized by hyperglycemia, metabolic acidosis, ketonemia, and ketonuria, which are caused by insulin deficiency and/or relatively reduced insulin effectiveness. The severity of DKA is categorized as mild to severe by the degree of metabolic acidosis, and morbidity and mortality are markedly increased in severe DKA." Among causes of morbidity and mortality in DKA, cerebral edema is the major cause, and rarely, many factors are included such as hypoglycemia, respiratory complications, sepsis, peripheral venous thrombosis, acute renal failure, acute pancreatitis, and other central nervous system complications. Electrolyte imbalance also frequently develops in DKA patients as low levels of the anabolic hormone insulin promote catabolic processes. Hyperglycemia-induced osmotic diuresis increases loss of electrolytes and minerals. Metabolic acidosis causes shifts of potassium, phosphorus, and magnesium from the intracellular to extracellular compartment. Extracellular fluid volume expansion and correction of acidosis during DKA treatment also reduce serum ion concentrations. Most patients with DKA require electrolyte replacement for imbalances. Most cases of hypophosphatemia during DKA treatment are mild and self-limited. Routine phosphate supplementation has shown no clinical benefit. ${ }^{2,3)}$ Nonetheless, supplementation should be initiated if hypophosphatemia is severe and associated with symptoms of metabolic encephalopathy, impaired myocardial contractibility, respiratory failure, dysphagia, ileus, hematologic abnormalities, and rhabdomyolysis. ${ }^{4,5)}$ We report here a case of severe hypophosphatemia associated with acute respiratory failure in an adolescent during DKA treatment. 


\section{Case report}

A 14-year-old female with symptoms of nausea, vomiting, and abdominal pain for 3 days was admitted to the local clinic with the diagnosis of acute viral gastroenteritis. Laboratory results showing hyperglycemia (glucose, $500 \mathrm{mg} / \mathrm{dL}$ ) and ketonuria strongly indicated DKA. The patient was transferred to our emergency unit because of worsening mental status despite initiation of therapy with intravenous fluid and shortacting insulin. The patient's medical history was unremarkable (her mother had type 2 diabetes mellitus). On admission, the patient was lethargic, severely dehydrated, and drowsy (Glasgow Coma Scale [GCS, eye-verbal-motor] $=14$ [3-5-6]). Vital signs indicated tachycardia (124 beats/min), tachypnea (40 breaths/ $\min )$, and hypothermia $\left(35^{\circ} \mathrm{C}\right)$. Blood pressure was in the normal range (106/56 $\mathrm{mmHg}$ ), body weight had decreased by $1.7 \mathrm{~kg}$ in the previous month to $48 \mathrm{~kg}$ (25th-50th percentile), and body mass index was $20 \mathrm{~kg} / \mathrm{m}^{2}$ (50th-75th percentile). Chest and abdominal $\mathrm{x}$-rays showed no specific abnormalities. Initial venous blood gas analysis indicated metabolic acidosis (pH, 6.92; $\mathrm{PCO}_{2}, 27 \mathrm{mmHg} ; \mathrm{PO}_{2}, 57 \mathrm{mmHg} ; \mathrm{HCO}_{3}, 5.6 \mathrm{mmol} /$ L). Serum chemistry and electrolyte tests revealed $569 \mathrm{mg} / \mathrm{dL}$ glucose, $133 \mathrm{mmol} / \mathrm{L}$ corrected sodium, $3.2 \mathrm{mmol} / \mathrm{L}$ potassium, $8.5 \mathrm{mg} / \mathrm{dL}$ corrected calcium, $2.1 \mathrm{mg} / \mathrm{dL}$ phosphate, $25.9 \mathrm{mg} /$ $\mathrm{dL}$ blood urea nitrogen, $0.57 \mathrm{mg} / \mathrm{dL}$ creatinine, and $0.6 \mathrm{mmol} / \mathrm{L}$ lactate. Urinalysis indicated the presence of ketones and glucose. Endocrinologic assessments showed $0.26 \mathrm{ng} / \mathrm{mL}$ C-peptide (normal range, $0.6-2.3 \mathrm{ng} / \mathrm{mL}$ ) and $15.7 \%$ glycosylated hemoglobin. Antiglutamic acid decarboxylase was positive (1.72 $\mathrm{U} / \mathrm{mL}$; range, $0-1 \mathrm{U} / \mathrm{mL}$ ), whereas anti-islet cell and anti-insulin antibodies were negative. These findings indicated a diagnosis of type 1 diabetes and severe DKA. The main metabolic data during hospitalization are shown in Table 1. Fluid $(0.45 \%$ saline) and continuous intravenous insulin infusion therapy were initiated at a rate of $0.05 \mathrm{unit} / \mathrm{kg} / \mathrm{hr}$ immediately according to DKA treatment protocol. ${ }^{5)}$ Hourly neurological evaluations were performed for signs of cerebral edema. Although the patient remained drowsy, signs of cerebral edema were not evident. The patient suffered $\mathrm{a}<1$ min generalized tonic seizure at 4 hours postadmission that was followed by bradycardia and cardiopulmonary arrest. External cardiac massage was initiated, followed by one epinephrine injection. Spontaneous circulation resumed after 6 minutes of cardiopulmonary resuscitation. Laboratory data after return of spontaneous circulation showed metabolic acidosis and hypophosphatemia ( $\mathrm{pH}, 7.05 ; \mathrm{PCO}_{2}, 23.6 \mathrm{mmHg} ; \mathrm{PO}_{2}, 181 \mathrm{mmHg} ; \mathrm{HCO}_{3}, 6.7$ $\mathrm{mmol} / \mathrm{L}$; glucose, $517 \mathrm{mg} / \mathrm{dL}$; corrected sodium, $136 \mathrm{mmol} /$ L; potassium, $3.2 \mathrm{mmol} / \mathrm{L}$; corrected calcium, $8.8 \mathrm{mg} / \mathrm{dL}$; phosphate, $1.2 \mathrm{mg} / \mathrm{dL}$ ). The patient received $\mathrm{K}_{2} \mathrm{HPO}_{4}$ and $\mathrm{KCl}$ to achieve $1.9 \mathrm{mEq} / \mathrm{kg} / \mathrm{day}$ of phosphorus after the cardiac arrest. A brain computed tomographic (CT) scan showed no brain edema or intracranial pathology. Dexamethasone and mannitol were prescribed for prevention of brain edema and the patient was admitted to the intensive care unit. Although the patient remained drowsy (GCS=14 [3-5-6]), vital signs were stable. At 16 hours after initiation of DKA treatment, the patient became stuporous (GCS=9 [2-2-5]) with shallow, weak, and slow respiration that necessitated endotracheal intubation. Blood tests at this time revealed respiratory acidosis and severe hypophosphatemia ( $\mathrm{pH}, 6.80 ; \mathrm{PCO}_{2}, 92.9 \mathrm{mmHg} ; \mathrm{PO}_{2}, 160.3$ $\mathrm{mmHg} ; \mathrm{HCO}_{3}, 14.6 \mathrm{mmol} / \mathrm{L}$; glucose, $375 \mathrm{mg} / \mathrm{dL}$; corrected sodium, $142 \mathrm{mmol} / \mathrm{L}$; potassium, $2.8 \mathrm{mmol} / \mathrm{L}$; phosphate, $0.4 \mathrm{mg} / \mathrm{dL}$; lactate, $0.6 \mathrm{mmol} / \mathrm{L}$ ). Phosphate replacement was increased to achieve a $3.8 \mathrm{mEq} / \mathrm{kg} /$ day phosphorus rate. A repeat brain CT imaging study showed no specific findings. The patient's electrolyte and $\mathrm{pH}$ values began normalizing within 2 days but mechanical ventilation was still required because hypophosphatemia was not alleviated (phosphate, $1.4 \mathrm{mg} / \mathrm{dL}$ ). Phosphate replacement was increased to achieve a $5.7 \mathrm{mEq} / \mathrm{kg} /$ day phosphorus rate. Fluid replacement with potassium and phosphate, and continuous intravenous insulin administration was maintained until laboratory findings were normal. On the third day, the patient's vital signs were stable, self-respiration was recovered, arterial blood gas levels were normal ( $\mathrm{pH}, 7.39$; $\mathrm{PCO}_{2}, 25.2 \mathrm{mmHg} ; \mathrm{PO}_{2}, 139.3 \mathrm{mmHg} ; \mathrm{HCO}_{3}, 15.6 \mathrm{mmol} / \mathrm{L}$ ), and plasma phosphate had risen $(3.5 \mathrm{mg} / \mathrm{dL})$. Weaning from inotropic and ventilator supports was possible. The patient was transferred to the general ward the next day. On the fourth day of hospitalization, oral feeding and multiple subcutaneous insulin injections were started and phosphate supplementation was stopped. An electroencephalogram showed no epileptiform discharges or slow waves. The patient was discharged on day 10

Table 1. Laboratory finding during admission

\begin{tabular}{|c|c|c|c|c|c|c|c|c|c|c|}
\hline Variable & Initial & $2 \mathrm{hr}$ & $4 \mathrm{hr}^{*}$ & $7 \mathrm{hr}$ & $11 \mathrm{hr}$ & $13 \mathrm{hr}$ & $16 \mathrm{hr}^{\dagger}$ & $18 \mathrm{hr}$ & 2 days & 3 days \\
\hline Glucose (70-110 mg/dL) & 569 & 521 & 517 & 416 & 400 & 417 & 375 & 321 & 352 & 241 \\
\hline pH value (7.35-7.45) & 6.92 & 7.03 & 7.05 & 7.07 & 7.09 & 7.13 & 6.80 & 7.18 & 7.28 & 7.39 \\
\hline Bicarbonate (21-28 mmol/L) & 5.6 & 4.2 & 6.7 & 5.2 & 7.2 & 9.0 & 14.6 & 8.8 & 11.3 & 15.6 \\
\hline Sodium (135-145 mmol/L) & 125 & 122 & 129 & 123 & 131 & 135 & 138 & 136 & 139 & 139 \\
\hline Corrected sodium (mmol/L) & 133 & 129 & 136 & 128 & 136 & 140 & 142 & 140 & 143 & 141 \\
\hline Potassium (3.5-5.5 mmol/L) & 3.2 & 3.6 & 3.2 & 4.3 & 3.0 & 2.6 & 2.8 & 3.4 & 2.8 & 5.1 \\
\hline Phosphate (3.9-5.8 mg/dL) & 2.1 & - & 1.2 & - & - & - & 0.4 & 0.8 & 1.4 & 3.5 \\
\hline Calcium (8.8-10.8 mg/dL) & 7.6 & - & 7.6 & 7.9 & - & - & 9.2 & 9.0 & 8.0 & 6.9 \\
\hline Magnesium (0.75-1.2 mmol/L) & - & - & - & 0.7 & - & - & - & 0.75 & - & 0.5 \\
\hline Albumin (3.8-5.4 g/dL) & 2.9 & - & 2.5 & - & - & - & 2.6 & 2.6 & 2.5 & 2.4 \\
\hline
\end{tabular}

*After return of spontaneous circulation. ${ }^{\dagger}$ Respiratory failure. 
with no neurologic complications. The patient was followed up regularly. No neurologic complications or respiratory problems were noted, and the patient did not require further phosphate supplementation.

This study approval and informed consent were waived by the Institutional Review Board of Severance Hospital.

\section{Discussion}

This case describes a patient who survived cardiac arrest and subsequent respiratory failure due to severe hypophosphatemia during treatment of DKA. The pathophysiologic mechanisms involved in the development of hypophosphatemia in DKA include osmotic diuresis, which is caused by glycosuria and results in urinary phosphate excretion, and metabolic acidosis, which shifts phosphate into the extracellular compartment. Hypophosphatemia may be worsened during treatment of DKA because insulin causes a shift of phosphate into the cellular compartment and fluid replacement dilutes the phosphate concentration. ${ }^{1)}$ These factors frequently lead to mild to moderate hypophosphatemia in DKA patients.

Severe hypophosphatemia causes the depletion of erythrocyte 2,3-diphosphoglycerate, which promotes impairment of oxygen delivery to peripheral tissues. The depletion of high-energy phosphate compounds in muscles leads to muscle weakness and rhabdomyolysis, dysrhythmias, myocardial dysfunction, and neurologic symptoms like seizures. ${ }^{6,7)}$ However, hypophosphatemia usually does not cause clinically significant symptoms and is acute and self-limited. Therefore, phosphate replacement in DKA treatment has been controversial, with prospective studies showing no clinical benefit. ${ }^{2,3,8)}$ Current guideline does not recommend routine phosphate replacement. ${ }^{5)}$ However, severe hypophosphatemia $(<1 \mathrm{mg} / \mathrm{dL})$ even in the absence of symptoms, or hypophosphatemia associated with metabolic encephalopathy, impaired myocardial contractibility, respiratory failure, muscle weakness, or rhabdomyolysis should be treated.

Cerebral edema should be taken into consideration in the differential diagnosis because the presented symptoms were difficult to distinguish from hypophosphatemia. The patient in this case presented with symptoms and signs suggesting cerebral edema, such as aggravated drowsiness, slowing of heart rate, and desaturation 4 hours after DKA treatment. However, brain CT scans at 2 different times revealed no abnormalities. Although there was gradual improvement in the patient's clinical condition after administration of treatment for DKA and cerebral edema, respiratory failure necessitating intubation developed within 16 hours. Respiratory failure in DKA can be caused by electrolyte imbalance (low levels of potassium, magnesium, and phosphate), cerebral edema, pulmonary edema, and infection. We considered severe hypophosphatemia as one of the important reasons for respiratory failure in this case because laboratory findings showed an initial abrupt decrease in phosphate level (from 2.1 to $0.4 \mathrm{mg} / \mathrm{dL}$ ) followed by symptom improvement after phosphate repletion treatment.
The normal brain CT scan findings support the hypothesis that hypophosphatemia was the cause of the respiratory complication. Previous case reports demonstrated that hypophosphatemia in DKA can be associated with respiratory failure, ${ }^{9-11)}$ cardiac arrest, ${ }^{12)}$ or seizure ${ }^{13)}$ encephalopathy, ${ }^{14)}$ or rhabdomyolysis. ${ }^{15)}$

Among the electrolytes that are monitored during DKA management, phosphate is easily overlooked. According to DKA protocol, potassium is recommended to be replaced with a concentration of $20 \mathrm{mmol} / \mathrm{L}$ as form of either potassium phosphate with potassium chloride or potassium acetate. ${ }^{5,16)}$ By replacing with potassium phosphate, phosphorus is also replaced. Although phosphorus is replaced simultaneously, the serum level of phosphate should require close monitoring. Especially, if the serum phosphate level is low or in the lower limit of normal range, phosphorus should be replaced more sufficiently. Severe hypophosphatemia in patients undergoing DKA treatment is rare but results in severe, though preventable, consequences. Although insulin and fluid replacement are the most important components of DKA treatment, early diagnosis and correction of electrolyte and blood chemistry imbalances should also be emphasized.

\section{Conflict of interest}

No potential conflict of interest relevant to this article was reported.

\section{References}

1. Wolfsdorf J, Craig ME, Daneman D, Dunger D, Edge J, Lee $\mathrm{W}$, et al. Diabetic ketoacidosis in children and adolescents with diabetes. Pediatr Diabetes 2009; 10 Suppl 12:118-33.

2. Wilson HK, Keuer SP, Lea AS, Boyd AE 3rd, Eknoyan G. Phosphate therapy in diabetic ketoacidosis. Arch Intern Med 1982;142:517-20.

3. Fisher JN, Kitabchi AE. A randomized study of phosphate therapy in the treatment of diabetic ketoacidosis. J Clin Endocrinol Metab 1983;57:177-80.

4. Gaasbeek A, Meinders AE. Hypophosphatemia: an update on its etiology and treatment. Am J Med 2005;118:1094101.

5. Wolfsdorf JI, Allgrove J, Craig ME, Edge J, Glaser N, Jain V, et al. ISPAD Clinical Practice Consensus Guidelines 2014. Diabetic ketoacidosis and hyperglycemic hyperosmolar state. Pediatr Diabetes 2014;15 Suppl 20:154-79.

6. Konstantinov NK, Rohrscheib M, Agaba EI, Dorin RI, Murata GH, Tzamaloukas AH. Respiratory failure in diabetic ketoacidosis. World J Diabetes 2015;6:1009-23.

7. Ditzel J, Lervang HH. Disturbance of inorganic phosphate metabolism in diabetes mellitus: clinical manifestations of phosphorus-depletion syndrome during recovery from diabetic ketoacidosis. Diabetes Metab Syndr Obes 2010;3:319-24. 
8. Gibby OM, Veale KE, Hayes TM, Jones JG, Wardrop CA. Oxygen availability from the blood and the effect of phosphate replacement on erythrocyte 2,3-diphosphoglycerate and haemoglobin-oxygen affinity in diabetic ketoacidosis. Diabetologia 1978;15:381-5.

9. Liu PY, Jeng CY. Severe hypophosphatemia in a patient with diabetic ketoacidosis and acute respiratory failure. J Chin Med Assoc 2004;67:355-9.

10. Spicek-Macan J, Hodoba N, Kolarić N, Nikolić I, MajerićKogler V, Popović-Grle S. Immeasurable levels of serum phosphate: an unidentified cause of respiratory failure in a diabetic patient. Coll Antropol 2010;34:1457-60.

11. Hasselstrøm L, Wimberley PD, Nielsen VG. Hypophosphatemia and acute respiratory failure in a diabetic patient. Intensive Care Med 1986;12:429-31.

12. Osuka A, Matsuoka T, Idoguchi K. Is this the worst outcome of metabolic syndrome? Hypophosphatemia and resulting cardiac arrest during the treatment of diabetic ketoacidosis with hypertriglyceridemia. Intern Med 2009;48:1391-5.

13. de Oliveira Iglesias SB, Pons Leite H, de Carvalho WB. Hypophosphatemia-induced seizure in a child with diabetic ketoacidosis. Pediatr Emerg Care 2009;25:859-61.

14. Mégarbane B, Guerrier G, Blancher A, Meas T, Guillausseau PJ, Baud FJ. A possible hypophosphatemia-induced, lifethreatening encephalopathy in diabetic ketoacidosis: a case report. Am J Med Sci 2007;333:384-6.

15. Shah SK, Shah L, Bhattarai S, Giri M. Rhabdomyolysis Due to severe hypophosphatemia in diabetic ketoacidosis. JNMA J Nepal Med Assoc 2015;53:137-40.

16. Svoren BM, Jospe N. Type 1 diabetes mellitus (immune mediated). In: Kliegman RM, Stanton B, St. Geme J, Schor N, Behrman RE. Nelson textbook of pediatrics. 20th ed. Philadelphia: Elsevier Saunders, 2016:2763-83. 